\title{
Somatic Incompatibility following Plasmodial Fusion between Strains of the Myxomycete Physarum polycephalum: the Effect on their Nuclei
}

\author{
By D. J. BORDER AND M. J. CARLILE \\ Department of Biochemistry, Imperial College of Science and Technology, \\ London $\mathrm{SW}_{7} 2 \mathrm{AZ}$
}

(Received I3 March 1974; revised 24 June 1974)

SUMMARY

Fusion of plasmodia of strains I5 and 29 of the myxomycete Physarum polycephalum under appropriate conditions results in a lethal reaction visible to the naked eye. Examination by autoradiography and electron microscopy of areas in which the reaction is occurring shows that most of the nuclei derived from strain 29 (the sensitive strain) are enclosed in vesicles and are disintegrating, but that almost all those originating from strain 15 (the killer) are healthy. At this stage other organelles appear to be undamaged. These results support the view that the essential feature of the reaction is the elimination of the nuclei of the sensitive strain and that the ultimate destruction of large areas which occurs under a limited range of experimental conditions is a secondary effect which may not occur in nature.

\section{INTRODUCTION}

Somatic incompatibility between strains of the myxomycete Physarum polycephalum Schweinitz takes the form (Carlile \& Dee, 1967) of either a failure of plasmodia of different strains to fuse or a post-fusion reaction. This reaction was first recognized as a lethal interaction which occurred on rich media and destroyed much of one or both plasmodia. Carlile (1972) described in detail the unilateral interaction between two strains, 15 and 29, in which I5 was the killer and 29 the sensitive strain. Under conditions of suboptimal nutrition the lethal reaction did not occur, but disappearance of strain 29 still took place. Particles similar in size to nuclei were seen in vacuoles of strain 15 shortly after lethal interaction and such particles were left on the agar surface as the plasmodium retreated. It was suggested that strain 15 had the ability to recognize and destroy nuclei of strain 29 and that the lethal effect, when it occurred, was a secondary consequence of the attack on the sensitive nuclei. Ultrastructural and autoradiographic studies designed to test this hypothesis have now been carried out.

\section{METHODS}

Strains and medium. The origin of strains 15 and 29 and the composition of the semidefined (SD) agar medium are given elsewhere (Carlile, 1972). Experiments were carried out at $24^{\circ} \mathrm{C}$ and aseptic procedures employed throughout.

Light microscope studies. Plasmodia were grown on about $25 \mathrm{ml} \mathrm{SD}$ agar medium in Petri dishes of $9 \mathrm{~cm}$ diameter and lethal reactions obtained by inoculating the two strains on to a single Petri dish (Carlile, 1972). An alternative procedure, used in control experi- 
ments for autoradiography, was to grow plasmodia in Petri dishes of $5 \mathrm{~cm}$ diameter. These contained SD agar to a depth of $2 \mathrm{~mm}$, prepared by adding the haem $(0.037 \mathrm{ml})$ to $3.7 \mathrm{ml}$ molten $\left(40^{\circ} \mathrm{C}\right)$ agar medium immediately before pouring. The lethal reaction was initiated by transferring a small piece of the plasmodium of strain 15 onto the plasmodium of strain 29 when the radius of the latter was about half that of the Petri dish, usually 2 days after inoculation. A disc of plasmodium with underlying agar was cut with a cork-borer ( $7 \mathrm{~mm}$ diam) from near the margin of strain $\mathrm{I} 5$, and placed gently on strain 29 near the margin of the plasmodium in an inverted position, i.e. so that the two plasmodia were in contact. This procedure did not harm the plasmodia and fusion between the strains soon occurred. Chin, Friedrich \& Berstein (1972) showed by autoradiography that with a similar technique plasmodium fusion occurred within $30 \mathrm{~min}$ and considerable protoplasmic mixing in $60 \mathrm{~min}$, so rapid fusion of the two strains may be expected. About $6 \mathrm{~h}$ after disc transfer, dead areas visible to the naked eye appeared and spread for a few hours, destroying much of the plasmodium of strain 29.

Plasmodia were fixed in situ with a $2 \cdot 5 \%$ (v/v) solution of glutaraldehyde (TAAB Laboratories, Reading) in $0.1 \mathrm{M}$-sodium cacodylate buffer, $\mathrm{pH} 7 \cdot 2$, for $\mathrm{I} h$ at room temperature and cut into strips (Io $\times 2 \mathrm{~mm}$ ). The strips were washed three times ( $\mathrm{I} \mathrm{h} \mathrm{each)} \mathrm{in} \mathrm{the} \mathrm{caco-}$ dylate buffer, post-fixed for $\mathrm{I} h$ in a $\mathrm{r} \%(\mathrm{w} / \mathrm{v})$ solution of osmium tetroxide in cacodylate buffer, washed for 90 min in the buffer, dehydrated through a graded series of ethanol solutions, infiltrated and flat-embedded in resin (Spurr, I969). Sections I and $2 \mu \mathrm{m}$ thick were cut on an ultramicrotome (LKB Ultratome) with glass knives, and transferred with a fine brush to drops of water on cleaned glass slides, which were then warmed to $60^{\circ} \mathrm{C}$ to allow the sections to flatten and attach firmly to the slides. Photographs were taken with a Leitz Ortholux microscope using phase contrast.

Electron microscopy. Plasmodia were grown on $25 \mathrm{ml} \mathrm{SD}$ agar medium and lethal reactions obtained by inoculating both strains into the same Petri dish. Fixation and embedding were carried out as for light microscopy. Silver sections were cut and stained with $2 \%$ (w/v) aqueous uranyl acetate, with lead citrate (Reynolds, 1963) or with both solutions, and examined with an A.E.I. EM $6 \mathrm{~B}$ electron microscope.

Autoradiography. $\left[6-{ }^{3} \mathrm{H}\right]$ thymidine with a specific activity of $10000 \mathrm{mCi} / \mathrm{mmol}$ was obtained as a sterile aqueous solution of activity $\mathrm{I} \mathrm{mCi} / \mathrm{ml}$ from the Radiochemical Centre, Amersham, Buckinghamshire. The thymidine solution ( $0.1 \mathrm{ml}$ ) was added to $3.7 \mathrm{ml}$ volumes of SD agar medium immediately before pouring into $5 \mathrm{~cm}$ diam Petri dishes, to give an activity of $27 \mu \mathrm{Ci} / \mathrm{ml}$. Plasmodia were labelled by being grown for several days on the agar medium containing tritiated thymidine. Lethal reactions were then initiated by disc transfer and the material fixed, sectioned and mounted as for light microscopy.

Sections mounted on slides were coated with Kodak AR Io stripping film (Rogers, 1967), dried and maintained at $4{ }^{\circ} \mathrm{C}$ in the dark. Samples were taken at two-day intervals, developed for $5 \mathrm{~min}$ at $18{ }^{\circ} \mathrm{C}$ in Kodak Digb developer, washed in distilled water, fixed in $30 \%(\mathrm{w} / \mathrm{v})$ sodium thiosulphate for Io min, washed and dried. Photographs were taken with the microscope focused on the emulsion layer, not on the specimen, so as to demonstrate silver grains which, depending upon the focusing, showed as bright or dark dots.

\section{RESULTS}

\section{Light microscopy}

Sections of plasmodia of strains 15 and 29 (Fig. I $a$ ) were similar in all respects. Plasmodia were penetrated by extensive channels communicating with the exterior and bounded by the 

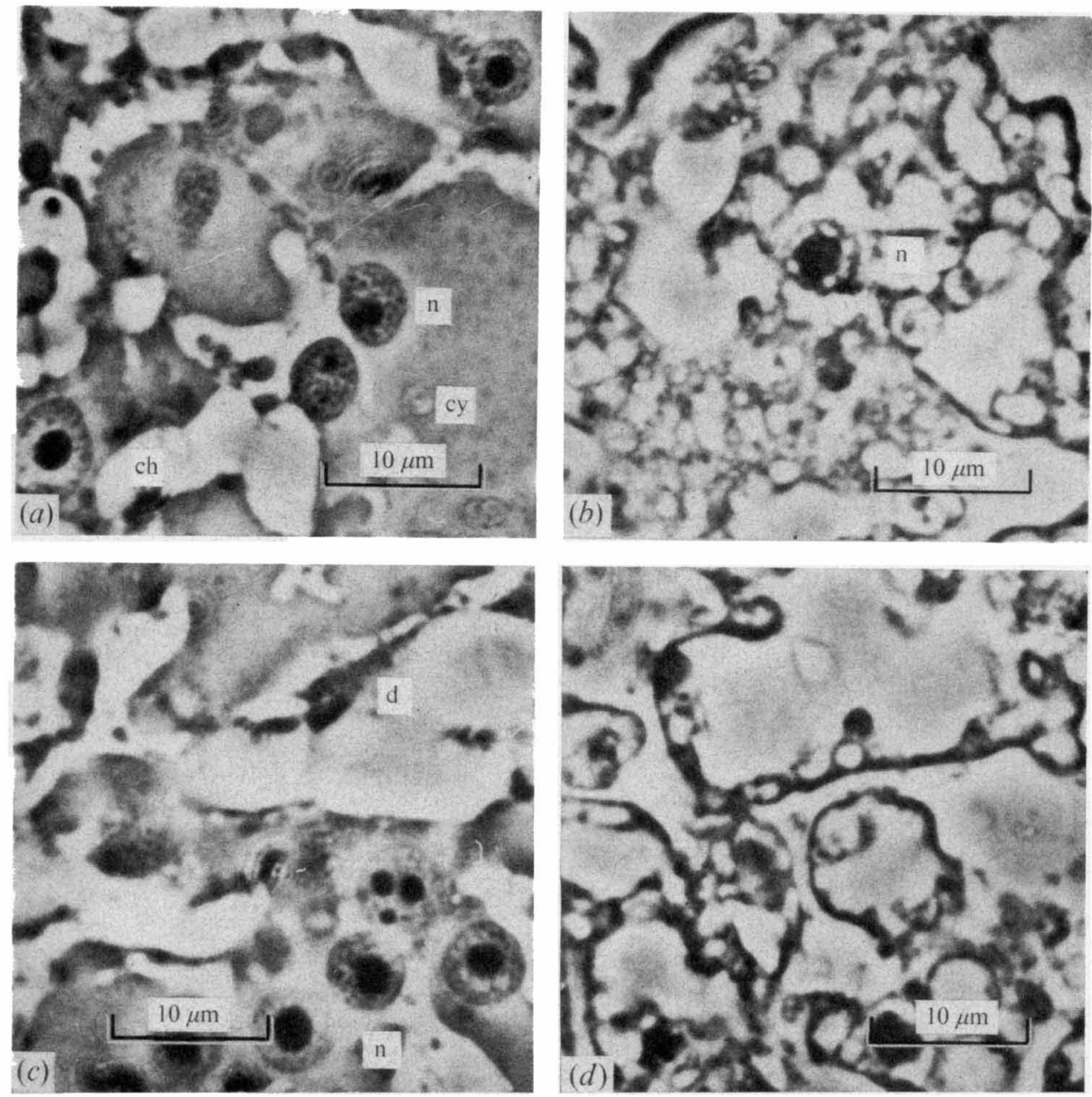

Fig. I. Phase-contrast photomicrographs of thin sections of unlabelled plasmodium. (a) Strain 29 showing healthy nuclei (n) with nucleoli and chromatin, cytoplasm (cy) with just perceptible mitochondria, and extensive channels (ch). (b) Dead area following lethal interaction of strains 15 and 29, showing refractile areas at the circumference of a nucleus (n). (c) A different region of the same dead area. Protoplasmic debris (d) is present in the channels, and apparently undamaged nuclei (n) in the cytoplasm. (d) Another region, showing complete disintegration.

plasmalemma (Rhea, I966; Daniel \& Järlfors, 1972). Nuclei contained prominent nucleoli and chromatin, and mitochondria were just detectable. Neither the plasmodia nor channels contained degenerate nuclei or debris.

Dead areas just visible to the naked eye appeared about $5 \mathrm{~h}$ after plasmodia of strains I 5 and 29 met and fused on an agar surface (Carlile, 1972). These areas spread rapidly and merged with each other giving a large dead area which approached maximum extent after a few hours, at which time fixation was carried out. Dead areas, although appearing uniform to the naked eye, were not strictly homogeneous since different regions were likely to differ both in the proportions of protoplasm contributed by the two strains and in the time elapsed since death. Differences in the degree of damage between different regions of a dead area were therefore to be expected, and were observed by light and electron microscopy. In 


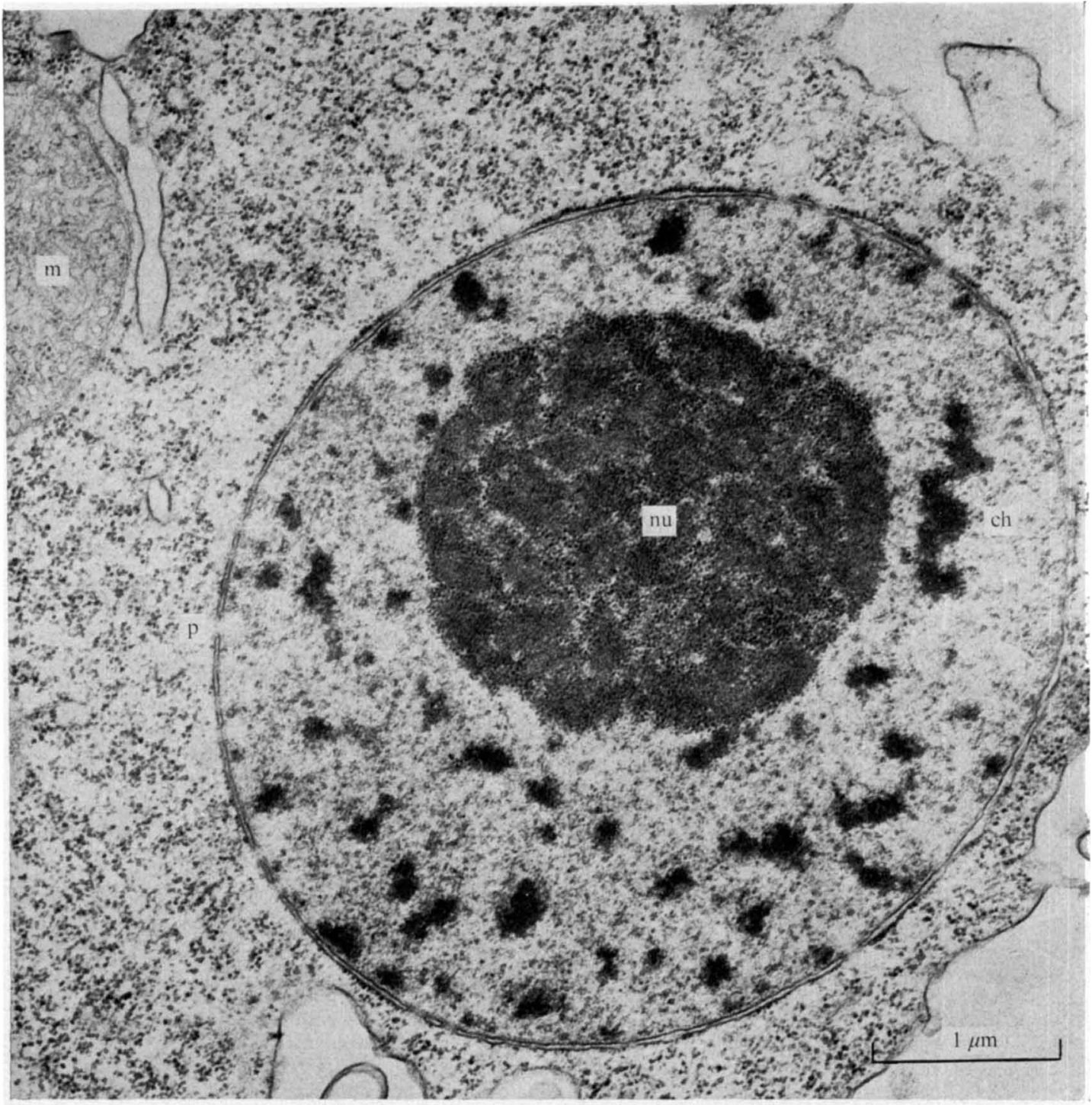

Fig. 2. Electron micrograph of an undamaged nucleus from a plasmodium undergoing a lethal reaction, showing nuclear membrane with pores (p) and chromatin (ch) including nucleolus (nu). The cytoplasm contains ribosomes and healthy mitochondria $(\mathrm{m})$.

some areas many nuclei were swollen to about twice their normal diameter and some had refractile areas around their circumference (Fig. I $b$ ). Extensive debris, consisting of damaged nuclei and cytoplasm and some crystalline material, occurred outside the plasmodium and in the extracellular channels, yet some of the nuclei within the plasmodium were apparently undamaged (Fig. I $c$ ). In other areas both nuclei and cytoplasm had completely disintegrated (Fig. I $d$ ). 


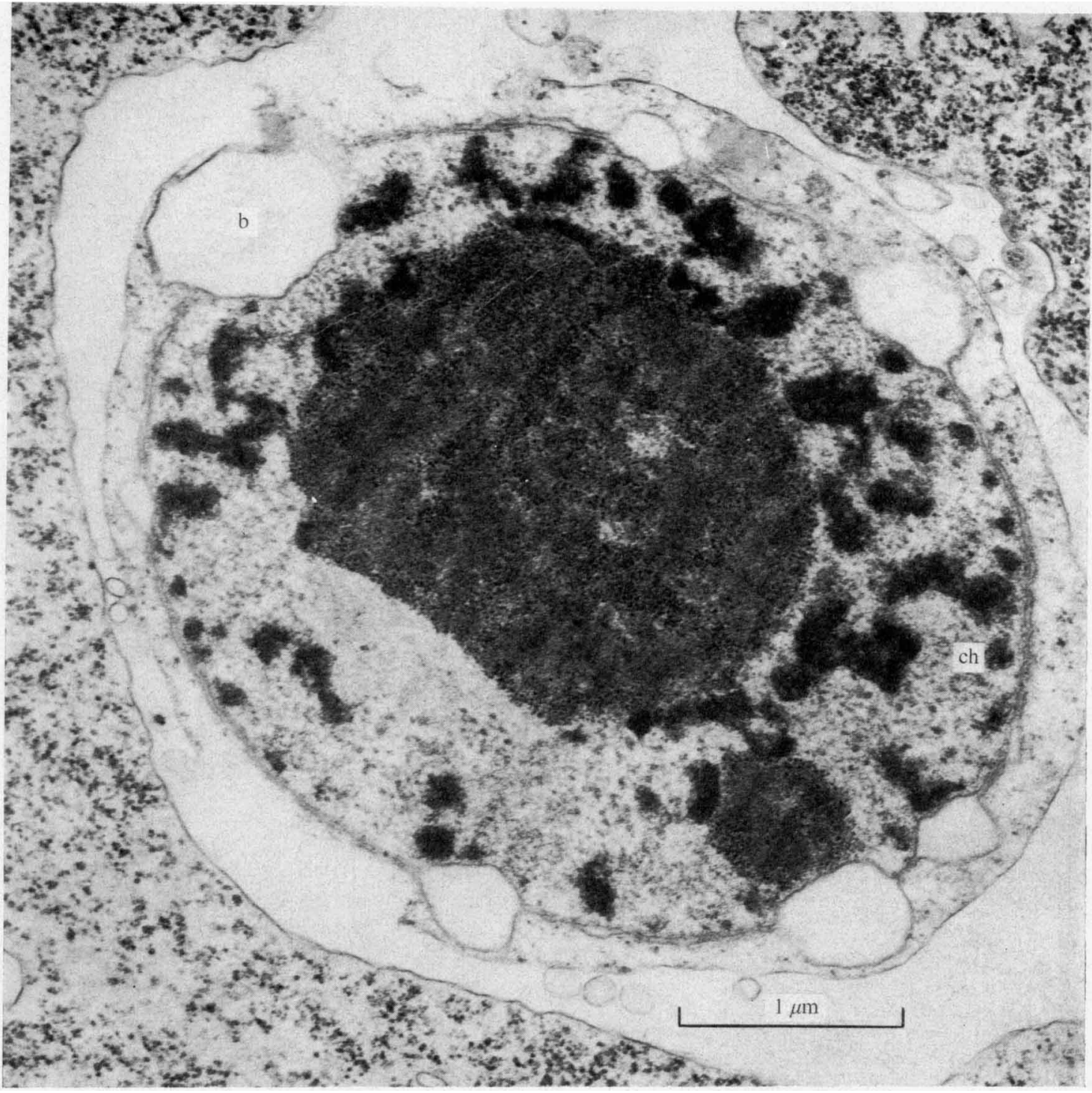

Fig. 3. A nucleus surrounded by a small amount of cytoplasm is enclosed in a vesicle. The double membrane surrounding the nucleus has separated at several points; these blebs (b) presumably correspond to the refractile points seen by phase-contrast microscopy (Fig. I $b$ ). Some clumping of chromatin (ch) and some nucleolar damage have occurred.

\section{Electron microscopy}

When examined by electron microscopy, strains 15 and 29 were similar to strains of $P$. polycephalum studied by other workers (Daniel \& Järlfors, 1972; Kessler, 1972; Rhea, 1966). Abnormal nuclei were not observed.

The dead areas produced by lethal interaction between the strains were heterogeneous when examined by electron microscopy, as was to be expected (see above). Undamaged nuclei (Fig. 2) and cytoplasm with apparently undamaged ribosomes and mitochondria were present in the vicinity of nuclei enclosed in vesicles and in various stages of disintegration (Figs. 3 to 5), although in some areas both nucleus and cytoplasm had disintegrated. 


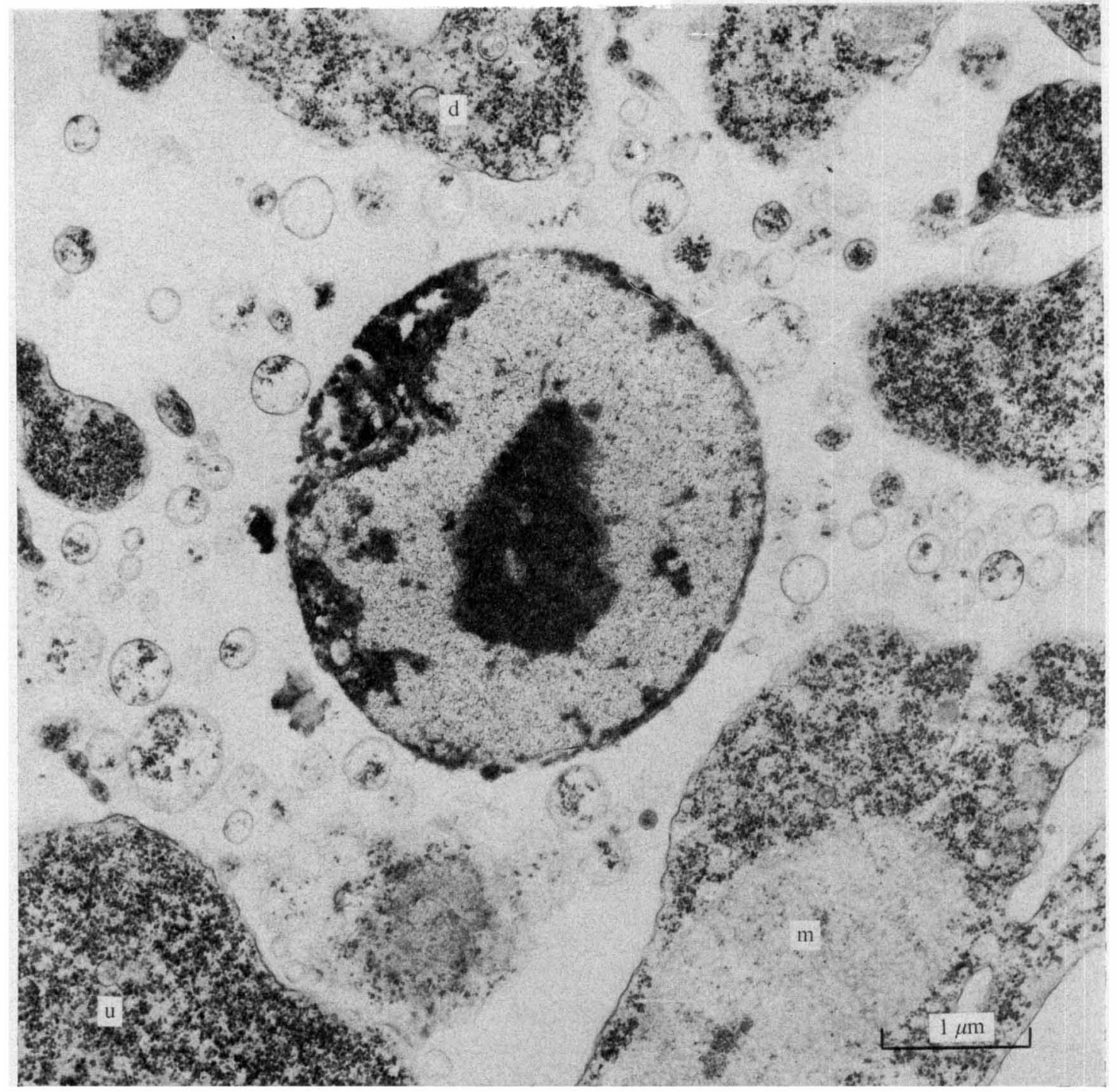

Fig. 4. A nucleus with cytoplasmic debris in a channel. The nuclear membrane has disappeared and the nucleolus and chromatin is damaged and distorted. Areas with undamaged $(\mathrm{u})$ and damaged (d) cytoplasm, including a damaged mitochondrion $(\mathrm{m})$ with disorganized lamellae are also present.

\section{Autoradiography}

Lethal interactions were carried out in which the nuclei of one strain were labelled with tritiated thymidine.

Discs were cut from a plasmodium of strain 15 that had been growing for several days on SD agar containing tritiated thymidine and were placed on a plasmodium of strain 29 growing on unlabelled SD agar. A lethal reaction occurred and the material was fixed. A comparable plasmodium of strain I 5 growing on labelled SD agar was also fixed. This was found to have normal nuclei of which 9I \% were labelled (Table I). In the reacted plasmodium, $82 \%$ of the unlabelled nuclei, which would mostly have been derived from strain 29 , showed damage but only $7 \%$ of the labelled nuclei, which would have originated from 


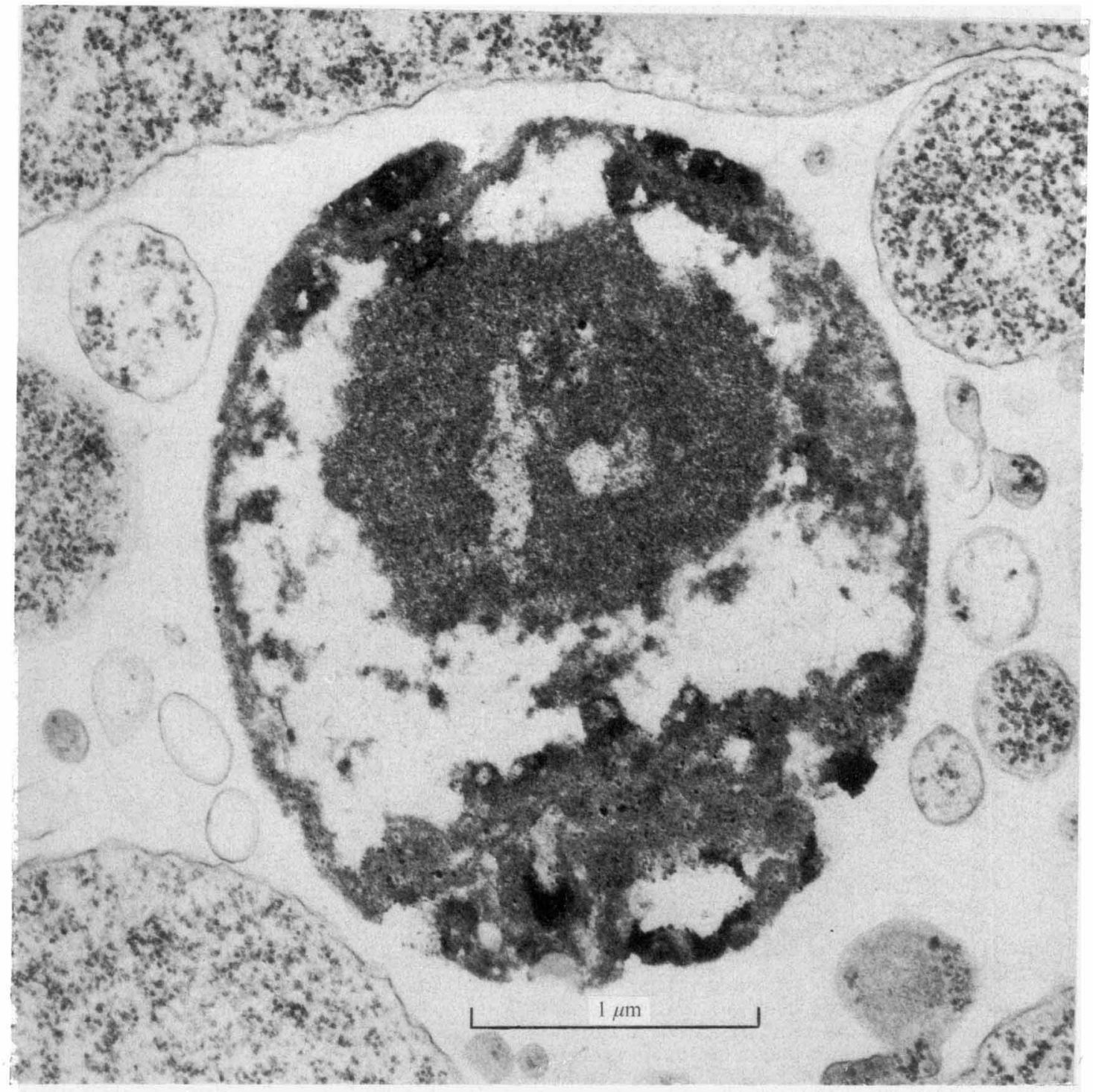

Fig. 5. A nucleus beginning to disintegrate, with leaching out of nuclear material.

strain I5, did. The normal appearance of a group of labelled nuclei in another experiment on the interaction of labelled strain 15 and unlabelled strain 29 is shown in Fig. 6(a).

A plasmodium of strain 29 was grown for several days on SD agar containing tritiated thymidine. A disc cut from a plasmodium of strain 15 growing on unlabelled medium was placed on the strain 29 plasmodium, a lethal reaction obtained, and the material fixed. A comparable plasmodium of strain 29 was also fixed and found to have $88 \%$ of the nuclei labelled; abnormal nuclei were not observed (Table I). Counts on an area of the reacted plasmodium distant from the lethal reaction showed $9 \mathrm{I} \%$ of the nuclei labelled and no abnormalities. At the site of the reaction $85 \%$ of the labelled nuclei, which would have been derived from strain 29 , were damaged but only $3 \%$ of the unlabelled ones were, most of which would have originated from strain 15 . A labelled swollen nucleus observed in a 
Table I. Frequency of labelled and damaged nuclei in autoradiographic experiments

I00 nuclei were examined in each control and 250 in the experimental plasmodia. A nucleus was recorded as labelled if one or more silver grains overlay the nucleus; commonly there were several. A nucleus was recorded as damaged if any trace of abnormality was observed.

\begin{tabular}{|c|c|c|c|c|c|c|}
\hline \multirow[b]{3}{*}{ Strain 15 labelled } & \multicolumn{2}{|c|}{ Labelled } & \multicolumn{2}{|c|}{ Unlabelled } & \multicolumn{2}{|c|}{ Damaged $*(\%)$} \\
\hline & Damaged & Normal & Damaged & Normal & 15 & 29 \\
\hline & & & & & & \\
\hline Control plasmodium & 0 & $9 I$ & 0 & 9 & 0 & - \\
\hline Lethal interaction & 7 & 92 & I 22 & 29 & 7 & 82 \\
\hline Strain 29 labelled & & & & & & \\
\hline Control plasmodium & 0 & 88 & 0 & 12 & - & 0 \\
\hline Control area & 0 & 228 & 0 & 22 & - & 0 \\
\hline Lethal interaction & 74 & I I & 5 & 160 & 3 & 85 \\
\hline
\end{tabular}

* The percentage of damaged nuclei among those presumed on the basis of labelling to be derived from the strain indicated.
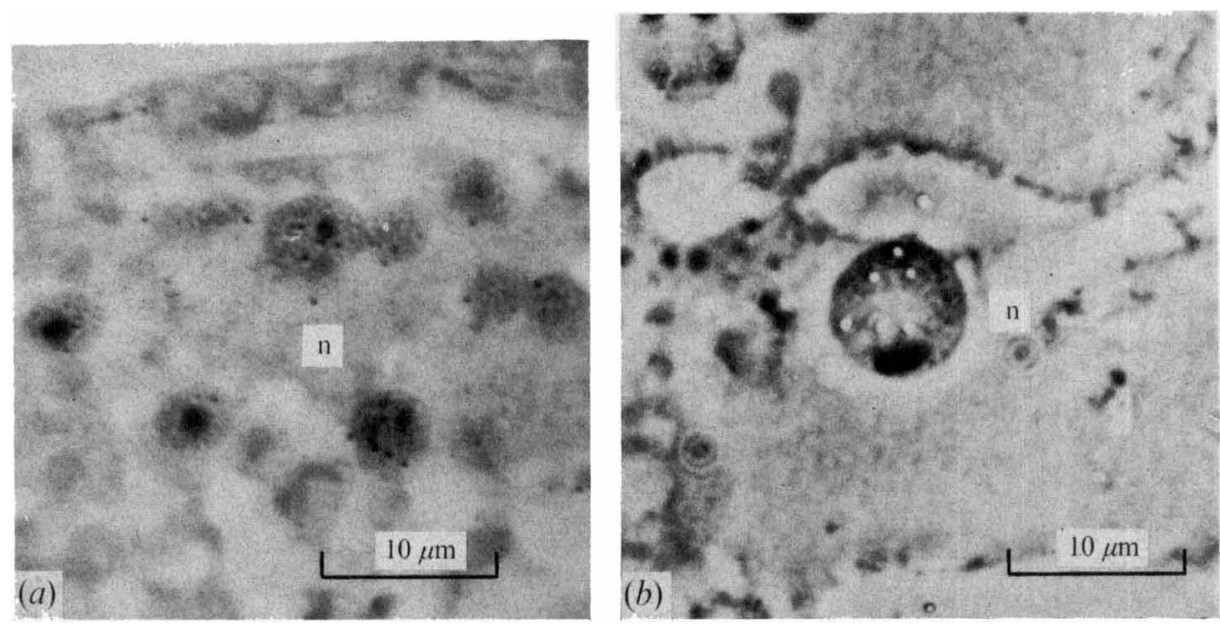

Fig. 6. Phase-contrast photomicrographs taken after lethal interaction between strains 15 and 29 , with one strain prelabelled with tritiated thymidine. The labelled strain was allowed to spread on unlabelled medium before the transfer was carried out. Silver grains show as bright or dark spots depending on plane of focus. (a) Strain I 5 prelabelled; a group of healthy nuclei ( $\mathrm{n}$ ) show labelling. (b) Strain 29 prelabelled; a swollen nucleus (n) shows labelling.

different experiment in which strain 29 was labelled is shown in Fig. 6(b); there was also much labelled ejected debris, which appeared to be derived from nuclei, and labelled nuclei in channels and vesicles.

The above experiments gave closely comparable results, over $80 \%$ of the nuclei deduced to originate from strain 29 being damaged and less than $10 \%$ of those likely to have come from strain 15. That some nuclei of strain 15 were damaged and some of strain 29 undamaged may be due to the limitations of the experimental method, since controls showed labelling to be about $90 \%$, with some fortuitous labelling occurring because of random distribution of silver grains. Alternatively, heterogeneity in the area of the reaction may be responsible, since at the time of fixation the reaction would have advanced at some points to the stage of death with the destruction of all organelles including nuclei of strain I5, whereas at other times even nuclei of strain 29 may not have shown damage. Also requiring 
comment is the relatively high frequency of nuclei presumed to be derived from strain 15 , $40 \%$ in one experiment and $66 \%$ in the other (Table I). A much lower frequency of strain I5 nuclei might have been expected because reactions were induced by placing a small portion of strain 15 on a much larger plasmodium of strain 29. However, since a massive ejection of debris resembling nuclei occurred before the appearance of a lethal reaction, and any ejected nuclei were likely to be derived from strain 29 , the proportion of nuclei derived from strain 15 would have increased by the time of fixation.

\section{DISCUSSION}

Fusion of plasmodia of strains 15 and 29 of Physarum polycephalum on plain agar leads to the disappearance of the features of strain 29 without any visible damage being observed. Under a range of other conditions the ejection of structures resembling nuclei is seen, the features of strain 29 disappear, but there is no striking lethal reaction (Carlile, 1972). These observations suggest that the essential feature of the post-fusion interaction between strains I5 and 29 is the elimination of the nuclei of strain 29 , and that the extensive lethal effects obtained in a limited range of laboratory conditions are a secondary consequence of the reactions that lead to the destruction of the nuclei and may not occur in nature (Carlile, I973).

Phase-contrast and electron microscope studies of fixed material of plasmodia undergoing the lethal reaction demonstrated the destruction of nuclei within vesicles. Autoradiography showed that these nuclei were mostly derived from the sensitive strain 29 . Thus a major feature of the unilateral lethal interaction between these two strains is the destruction of the nuclei of the sensitive strain.

\section{REFERENCES}

CARLILE, M. J. (1972). The lethal interaction following plasmodial fusion between two strains of the myxomycete Physarum polycephalum. Journal of General Microbiology 7I, 58I-590.

CARLILE, M. J. (1973). Cell fusion and somatic incompatibility in myxomycetes. Berichte der Deutschen Botanischen Gesellschaft 86, I23-I 39.

CarliLe, M. J. \& DeE, J. (1967). Plasmodial fusion and lethal interaction between strains in a myxomycete. Nature, London 215, 832-834.

Chin, B., Friedrich, P. D. \& Berstein, I. A. (1972). Stimulation of mitosis following fusion of plasmodia in the myxomycete Physarum polycephalum. Journal of General Microbiology 71, 93-IOI.

DANIEL, J. W. \& JÄrLFORS, U. (1972). Plasmodial ultrastructure of the myxomycete Physarum polycephalum. Tissue and Cell 4, $15-36$.

KessLer, D. (1972). On the location of myosin in the myxomycete Physarum polycephalum and its possible function in cytoplasmic streaming. Journal of Mechanochemistry and Cell Motility I, 125-137.

ReYNolds, E. S. (1963). The use of lead citrate at high $\mathrm{pH}$ as an electron-opaque stain in electron microscopy. Journal of Cell Biology 17, 208-212.

RHEA, R. P. (1966). Electron microscope observations on the slime mould Physarum polycephalum with specific reference to fibrillar structures. Journal of Ultrastructure Research 15, 349-379.

RogERs, A. W. (1967). Techniques of Autoradiography. Amsterdam: Elsevier.

SPURR, A. R. (1969). A low-viscosity epoxy resin embedding medium for electron microscopy. Journal of Ultrastructure Research 26, 3 I-43. 